\title{
Verification of ISS, iISS and IOSS properties applying weighted homogeneity
}

\author{
Emmanuel Bernuau, Andrey Polyakov, Denis Efimov and Wilfrid Perruquetti
}

\begin{abstract}
Several conditions are proposed to check different robustness properties (ISS, iISS, IOSS and OSS) for generic nonlinear systems applying the weighted homogeneity concept (global or local). The advantages of this result is that, under some mild conditions, the system robustness can be established as a function of the degree of homogeneity.
\end{abstract}

\section{INTRODUCTION}

The problem of robustness and stability analysis with respect to external inputs (like exogenous disturbances or measurement noises) for dynamical systems is in the center of attention of many research works [1], [2], [3], [4], [5], [6]. One of the most popular theories, which can be used for this robustness analysis for nonlinear systems, was originated more than 20 years ago [7] and it is based on the Input-to-State Stability (ISS) property and many related notions, see a recent survey [8] and the references therein. The advantages of ISS theory include a complete list of necessary and sufficient conditions, existence of the Lyapunov method extension, a rich variety of stability concepts adopted for different control and estimation problems.

The main tool to check the ISS property for a nonlinear system consists in a Lyapunov function design satisfying sufficient conditions. As usual, there is no generic approach to select a Lyapunov function for nonlinear systems. Therefore, computationally tractable approaches for ISS verification for particular classes of nonlinear systems are of great importance, and they are highly demanded in applications. In this work we are going to propose such a technique for checking ISS and other related properties (like integral ISS or input-output-to-state stability) for a class of homogeneous and locally homogeneous systems.

Homogeneity is an intrinsic property of an object, which remains consistent with respect to some scaling, e.g. level sets (resp. solutions) are preserved for homogeneous functions (resp. vector fields). The notion of weighted homogeneity was found useful by many authors [9], [10], [11], [12], [13], [14], [15], [16], [17]. The main feature of this property is that it transforms a local (stability) property of the system to the whole state space via a suitably defined scaling. In some cases such a globality of the system behavior becomes ambiguous, that is why the local homogeneity notion has been proposed [9], [18], [19]. In this case the property transfer can be carried out on a subspace using different local scales.

The ISS notion of homogeneous systems has been studied in [20], [21], [18]. In this work we are going to generalize the result of those works and extend it to the integral ISS (iISS) property and some other related notions. The underlying idea of the proposed results is that for a nonlinear system its asymptotic stability with zero disturbance implies a certain robustness (ISS or iISS) under homogeneity conditions. Note that to establish asymptotic stability of a homogeneous system one can use a non-strict Lyapunov function with the Krasovskii-LaSalle arguments.

The outline of the paper is as follows. Notations used in the paper are given in Section II. The robust stability notions under consideration and homogeneity are introduced in Section III. The ISS and iISS properties of homogeneous systems are studied in Section IV. The same analysis for locally homogeneous systems is done in Section V. An extension to some robust stability properties for the systems with an output is considered in Section VI.

The first and the last two authors are with LAGIS UMR 8219, Université Lille Nord de France, Ecole Centrale de Lille, Avenue Paul Langevin, BP 48, 59651 Villeneuve d'Ascq, France. The last three authors are with Non-A project at Inria Lille, Parc Scientifique de la Haute Borne, 40 avenue Halley, 59650 Villeneuve d'Ascq, France. The third author is with Department of Control Systems and Informatics, National Research University ITMO, 49 Kronverkskiy av., Saint Petersburg, 197101, Russia. 


\section{NOTATION}

Through the paper the following notation is used:

- $\mathbb{R}_{+}=\{x \in \mathbb{R}: x \geq 0\}$, where $\mathbb{R}$ is the set of real number.

- $|\cdot|$ denotes the absolute value in $\mathbb{R},\|\cdot\|$ denotes the Euclidean norm on $\mathbb{R}^{n}$.

- For a (Lebesgue) measurable function $d: \mathbb{R}_{+} \rightarrow \mathbb{R}^{m}$ define the norm $\|d\|_{\left[t_{0}, t_{1}\right)}=\operatorname{ess} \sup _{t \in\left[t_{0}, t_{1}\right)}\|d(t)\|$, then $\|d\|_{\infty}=$ $\|d\|_{[0,+\infty)}$ and the set of $d(t)$ with the property $\|d\|_{\infty}<+\infty$ we further denote as $\mathcal{L}_{\infty}$ (the set of essentially bounded measurable functions).

- A continuous function $\alpha: \mathbb{R}_{+} \rightarrow \mathbb{R}_{+}$belongs to the class $\mathcal{K}$ if $\alpha(0)=0$ and the function is strictly increasing. The function $\alpha: \mathbb{R}_{+} \rightarrow \mathbb{R}_{+}$belongs to the class $\mathcal{K}_{\infty}$ if $\alpha \in \mathcal{K}$ and it is increasing to infinity. A continuous function $\beta: \mathbb{R}_{+} \times \mathbb{R}_{+} \rightarrow \mathbb{R}_{+}$belongs to the class $\mathcal{K} \mathcal{L}$ if $\beta(\cdot, t) \in \mathcal{K}_{\infty}$ for each fixed $t \in \mathbb{R}_{+}$and $\lim _{t \rightarrow+\infty} \beta(s, t)=0$ for each fixed $s \in \mathbb{R}_{+}$. For any $\alpha \in \mathcal{K}$ and $s, r \in \mathbb{R}_{+}, \alpha(s+r) \leq \alpha(2 s)+\alpha(2 r)$.

- The notation $D V(x) f(x)$ stands for the directional derivative of a continuously differentiable function $V$ with respect to the vector field $f$ evaluated at point $x$.

- Young's inequality: $s v \leq \frac{s^{p}}{p}+\left(1-\frac{1}{p}\right) v^{\frac{p}{p-1}}$ for any $s, v \in \mathbb{R}_{+}$and $p>1$.

- A series of integers $1,2, \ldots, n$ is denoted by $\overline{1, n}$.

\section{PRELIMINARIES}

In this work the following nonlinear system is considered:

$$
\dot{x}=f(x, d),
$$

where $x \in \mathbb{R}^{n}$ is the state, $d \in \mathbb{R}^{m}$ is the external input, $d(t) \in \mathcal{L}_{\infty}$, and $f: \mathbb{R}^{n+m} \rightarrow \mathbb{R}^{n}$ is a locally Lipschitz (or Hölder) continuous function, $f(0,0)=0$. In some cases the system (1) is equipped with an output $y \in \mathbb{R}^{p}$ :

$$
y=h(x),
$$

where $h: \mathbb{R}^{n} \rightarrow \mathbb{R}^{p}$ is a continuous function. For an initial condition $x_{0} \in \mathbb{R}^{n}$ and input $d \in \mathcal{L}_{\infty}$, define the corresponding solutions by $x\left(t, x_{0}, d\right)$ for any $t \geq 0$ for which the solution exists.

\section{A. Stability properties}

In this work we will be interested in the following stability properties [8].

Definition 1. The system (1) is called input-to-state practically stable (ISpS), if for any input $d \in \mathcal{L}_{\infty}$ and any $x_{0} \in \mathbb{R}^{n}$ there are some functions $\beta \in \mathcal{K} \mathcal{L}, \gamma \in \mathcal{K}$ and $c \geq 0$ such that

$$
\left\|x\left(t, x_{0}, d\right)\right\| \leq \beta\left(\left\|x_{0}\right\|, t\right)+\gamma\left(\|d\|_{[0, t)}\right)+c \quad \forall t \geq 0 .
$$

The function $\gamma$ is called nonlinear asymptotic gain. The system is called ISS if $c=0$.

Definition 2. The system (1) is called iISS, if there are some functions $\alpha \in \mathcal{K}_{\infty}, \gamma \in \mathcal{K}$ and $\beta \in \mathcal{K} \mathcal{L}$ such that for any $x_{0} \in \mathbb{R}^{n}$ and $d \in \mathcal{L}_{\infty}$ the estimate holds:

$$
\alpha\left(\left\|x\left(t, x_{0}, d\right)\right\|\right) \leq \beta\left(\left\|x_{0}\right\|, t\right)+\int_{0}^{t} \gamma(\|d(s)\|) d s \quad \forall t \geq 0 .
$$

Definition 3. The system (1), (2) with $d=0$ is called Output-to-State Stable (OSS), if there are functions $\beta \in \mathcal{K} \mathcal{L}$ and $\gamma \in \mathcal{K}$ such that for all $x_{0} \in \mathbb{R}^{n}$

$$
\left\|x\left(t, x_{0}, 0\right)\right\| \leq \beta\left(\left\|x_{0}\right\|, t\right)+\gamma\left(\|y\|_{[0, t)}\right) \quad \forall t \in\left[0, t_{\max }\left(x_{0}\right)\right) .
$$

In this definition $t_{\max }\left(x_{0}\right) \leq+\infty$ determines the interval of solutions existence for the system $(1),(2) ; y(t)=h\left[x\left(t, x_{0}, 0\right)\right]$ is the output solution. 
Definition 4. The system (1), (2) is called input-output-to-state stable (IOSS) if there are functions $\beta \in \mathcal{K} \mathcal{L}$ and $\gamma_{1}, \gamma_{2} \in \mathcal{K}$ such that for all $x_{0} \in \mathbb{R}^{n}, d \in \mathcal{L}_{\infty}$ and all $t \in\left[0, t_{\max }\left(x_{0}, d\right)\right)$,

$$
\left\|x\left(t, x_{0}, d\right)\right\| \leq \beta\left(\left\|x_{0}\right\|, t\right)+\gamma_{1}\left(\|d\|_{[0, t)}\right)+\gamma_{2}\left(\|y\|_{[0, t)}\right) .
$$

Where again $t_{\max }\left(x_{0}, d\right) \leq+\infty$ defines the interval of existence of the system (1), (2) solutions, $y(t)=h\left[x\left(t, x_{0}, d\right)\right]$ is the corresponding output solution.

These properties have the following Lyapunov function characterizations.

Definition 5. A smooth function $V: \mathbb{R}^{n} \rightarrow \mathbb{R}_{+}$is called ISpS Lyapunov function for the system (1) if for all $x \in \mathbb{R}^{n}, d \in \mathbb{R}^{m}$ and some $r \geq 0, \alpha_{1}, \alpha_{2}, \alpha_{3} \in \mathcal{K}_{\infty}$ and $\theta \in \mathcal{K}$ :

$$
\begin{aligned}
\alpha_{1}(\|x\|) & \leq V(x) \leq \alpha_{2}(\|x\|), \\
D V(x) f(x, d) & \leq r+\theta(\|d\|)-\alpha_{3}(\|x\|) .
\end{aligned}
$$

Such a function $V$ is called ISS Lyapunov function if $r=0$, and it is iISS Lyapunov function if instead $\alpha_{3}: \mathbb{R}_{+} \rightarrow \mathbb{R}_{+}$is a positive definite function.

Note that an ISS Lyapunov function can also satisfy the following equivalent condition for some $\chi \in \mathcal{K}_{\infty}$ :

$$
\|x\|>\chi(\|d\|) \Rightarrow D V(x) f(x, d) \leq-\alpha_{3}(\|x\|)
$$

Definition 6. A smooth function $V: \mathbb{R}^{n} \rightarrow \mathbb{R}_{+}$is called OSS Lyapunov function for the system (1), (2) with $d=0$ if for all $x \in \mathbb{R}^{n}$ there are functions $\alpha_{1}, \alpha_{2}, \alpha_{3} \in \mathcal{K}_{\infty}, \sigma \in \mathcal{K}$ such that:

$$
\begin{gathered}
\alpha_{1}(\|x\|) \leq V(x) \leq \alpha_{2}(\|x\|), \\
D V(x) f(x, 0) \leq-\alpha_{3}(\|x\|)+\sigma(\|y\|) .
\end{gathered}
$$

Definition 7. A smooth function $V$ is called IOSS Lyapunov function for the system (1), (2) if for all $x \in \mathbb{R}^{n}, d \in \mathbb{R}^{m}$ and some functions $\alpha_{1}, \alpha_{2}, \alpha_{3} \in \mathcal{K}_{\infty}, \sigma_{1}, \sigma_{2} \in \mathcal{K}$ the inequalities hold:

$$
\begin{gathered}
\alpha_{1}(\|x\|) \leq V(x) \leq \alpha_{2}(\|x\|), \\
D V(x) f(x, d) \leq-\alpha_{3}(\|x\|)+\sigma_{1}(\|d\|)+\sigma_{2}(\|y\|) .
\end{gathered}
$$

Theorem 1. The system (1) is ISS (ISpS, iISS) iff it admits an ISS (ISpS, iISS) Lyapunov function.

Theorem 2. The system (1), (2) is IOSS (OSS for $d=0)$ iff it admits an IOSS (OSS) Lyapunov function.

Note that if the system (1) is ISS, then it is also iISS.

\section{B. Weighted homogeneity}

Following [22], for fixed strictly positive numbers $r_{i}, i=\overline{1, n}$ called weights and $\lambda>0$, one can define:

- the vector of weights $\mathbf{r}=\left(r_{1}, \ldots, r_{n}\right)^{T}, r_{\max }=\max _{1 \leq j \leq n} r_{j}$ and $r_{\min }=\min _{1 \leq j \leq n} r_{j}$;

- the dilation matrix function $\Lambda_{r}(\lambda)=\operatorname{diag}\left\{\lambda^{r_{i}}\right\}_{i=1}^{n}$, note that $\forall x \in \mathbb{R}^{n}$ and $\forall \lambda>0$ we have $\Lambda_{r}(\lambda) x=$ $\left(\lambda^{r_{1}} x_{1}, \ldots, \lambda^{r_{i}} x_{i}, \ldots, \lambda^{r_{n}} x_{n}\right)^{T}$

- the r-homogeneous norm $\|x\|_{r}=\left(\sum_{i=1}^{n}\left|x_{i}\right|^{\frac{\rho}{r_{i}}}\right)^{\frac{1}{\rho}}$ for any $x \in \mathbb{R}^{n}$ and $\rho=\Pi_{i=1}^{n} r_{i}$;

- the unit sphere in the homogeneous norm $S_{r}=\left\{x \in \mathbb{R}^{n}:\|x\|_{r}=1\right\}$.

Definition 8. A function $g: \mathbb{R}^{n} \rightarrow \mathbb{R}$ is $\mathbf{r}$-homogeneous with degree $\mu \in \mathbb{R}$ if $\forall x \in \mathbb{R}^{n}$ and $\forall \lambda>0$ we have:

$$
\lambda^{-\mu} g\left(\Lambda_{r}(\lambda) x\right)=g(x) .
$$

A vector field $f: \mathbb{R}^{n} \rightarrow \mathbb{R}^{n}$ is $\mathbf{r}$-homogeneous with degree $\nu \in \mathbb{R}$, with $\nu \geq-r_{\min }$ if $\forall x \in \mathbb{R}^{n}$ and $\forall \lambda>0$ we have:

$$
\lambda^{-\nu} \Lambda_{r}^{-1}(\lambda) f\left(\Lambda_{r}(\lambda) x\right)=f(x)
$$


which is equivalent for $i$-th component of $f$ being a $\mathbf{r}$-homogeneous function of degree $r_{i}+\nu$.

The system (1) with $d=0$ is $\mathbf{r}$-homogeneous of degree $\nu$ if the vector field $f$ is $\mathbf{r}$-homogeneous of degree $\nu$.

Theorem 3. [11], [9] For the system (1) with $d=0$ and $\mathbf{r}$-homogeneous and continuous function $f$ the following properties are equivalent:

- the system (1) is (locally) asymptotically stable;

- there exists a continuously differentiable $\mathbf{r}$-homogeneous Lyapunov function $V: \mathbb{R}^{n} \rightarrow \mathbb{R}_{+}$such that

$$
\begin{gathered}
\alpha_{1}(\|x\|) \leq V(x) \leq \alpha_{2}(\|x\|), D V(x) f(x, 0) \leq-\alpha(\|x\|), \\
\lambda^{-\mu} V\left(\Lambda_{r}(\lambda) x\right)=V(x), \mu>r_{\max }
\end{gathered}
$$

$\forall x \in \mathbb{R}^{n}$ and $\forall \lambda>0$, for some $\alpha_{1}, \alpha_{2} \in \mathcal{K}_{\infty}$ and $\alpha \in \mathcal{K}$.

The $\mathbf{r}$-homogeneity concept presented in Definition 8 is introduced for some $\mathbf{r}$ and all $\lambda>0$. Restricting the set of admissible values for $\lambda$ we can introduce local homogeneity [18], [19], [9].

Definition 9. A function $g: \mathbb{R}^{n} \rightarrow \mathbb{R}$ is $\left(\mathbf{r}_{0}, \lambda_{0}, g_{0}\right)$-homogeneous with degree $\nu_{0} \in \mathbb{R}\left(g_{0}\right.$ is a $\mathbf{r}_{0}$-homogeneous function and $\left.\lambda_{0} \in \mathbb{R}_{+} \cup\{+\infty\}\right)$ if for all $x \in S_{r_{0}}$ we have:

$$
\lim _{\lambda \rightarrow \lambda_{0}}\left(\lambda^{-\nu_{0}} g\left(\Lambda_{r_{0}}(\lambda) x\right)-g_{0}(x)\right)=0 .
$$

A vector field $f: \mathbb{R}^{n} \rightarrow \mathbb{R}^{n}$ is $\left(\mathbf{r}_{0}, \lambda_{0}, f_{0}\right)$-homogeneous with degree $\mu_{0} \geq-r_{0 \text { min }}\left(f_{0}\right.$ is a $\mathbf{r}_{0}$-homogeneous vector field and $\lambda_{0} \in \mathbb{R}_{+} \cup\{+\infty\}$ ) if for all $x \in S_{r_{0}}$ we have:

$$
\lim _{\lambda \rightarrow \lambda_{0}}\left(\lambda^{-\mu_{0}} \Lambda_{r_{0}}^{-1}(\lambda) f\left(\Lambda_{r_{0}}(\lambda) x\right)-f_{0}(x)\right)=0 .
$$

The limits have to be calculated uniformly on $S_{r_{0}}$ for $\lambda_{0} \in\{0,+\infty\}$.

The system (1) for $d=0$ is $\left(\mathbf{r}_{0}, \lambda_{0}, f_{0}\right)$-homogeneous with degree $\mu_{0} \in \mathbb{R}$ if the vector field $f$ is $\left(\mathbf{r}_{0}, \lambda_{0}, f_{0}\right)$-homogeneous with degree $\mu_{0}$.

The coefficients $r_{0 i}>0, i \in \overline{1, n}$ are called weights, $\nu_{0}$ (respectively $\mu_{0}$ ) is the degree of homogeneity (it may depend on $\lambda_{0}$ ) and $g_{0}$ (respectively $f_{0}$ ) is the approximating function of $g$ (respectively $f$ ) at $\lambda_{0}$.

Examples of locally homogeneous systems can be found in [18], [19].

Theorem 4. [23], [11], [9] Let the system (1) with $d=0$ be $\left(\mathbf{r}, 0, f_{0}\right)$-homogeneous with a continuous $f_{0}: \mathbb{R}^{n} \rightarrow \mathbb{R}^{n}$. If the system $\dot{x}=f_{0}(x)$ is (locally) asymptotically stable, then the system (1) is also locally asymptotically stable.

Theorem 5. [18], [9] Let the system (1) with $d=0$ be $\left(\mathbf{r},+\infty, f_{\infty}\right)$-homogeneous with a continuous $f_{\infty}: \mathbb{R}^{n} \rightarrow \mathbb{R}^{n}$. If the system $\dot{x}=f_{\infty}(x)$ is (globally) asymptotically stable, then there exists a compact invariant set $X_{\infty} \subset \mathbb{R}^{n}$ containing the origin such that the system (1) is globally asymptotically stable with respect to the set $X_{\infty}$.

Lemma 1. Let a function $g: \mathbb{R}^{n} \rightarrow \mathbb{R}$ be $\left(\mathbf{r},+\infty, g_{\infty}\right)$-homogeneous with degree $\nu, g$ and $g_{\infty}$ be continuous functions, then for all $x \in \mathbb{R}^{n}$

$$
\left|g(x)-g_{\infty}(x)\right| \leq \omega\left(\|x\|_{r}\right), \omega(s)=\left\{\begin{array}{ll}
k s^{w_{\min }} & \text { if } s \leq 1 \\
k s^{w_{\max }} & \text { if } s>1
\end{array},\right.
$$

where $k>0$ and $0 \leq w_{\min } \leq \nu, 0 \leq w_{\max }<\nu$

Proof: By definition for any $x \in \mathbb{R}^{n}$ there are $y \in S_{r}$ and $\lambda=\|x\|_{r}$ such that $x=\Lambda_{r}(\lambda) y$, then $\left|g(x)-g_{\infty}(x)\right|=$ $\left|g\left(\Lambda_{r}(\lambda) y\right)-g_{\infty}\left(\Lambda_{r}(\lambda) y\right)\right|$. For any $y \in S_{r}$ consider

$$
\left|\lambda^{-\nu} g\left(\Lambda_{r}(\lambda) y\right)-g_{\infty}(y)\right|=\lambda^{-\nu}\left|g\left(\Lambda_{r}(\lambda) y\right)-g_{\infty}\left(\Lambda_{r}(\lambda) y\right)\right|
$$

For $\lambda \rightarrow+\infty$ the left hand side of the relation above converges to zero, therefore there is $\bar{k}>0$ such that $\mid g\left(\Lambda_{r}(\lambda) y\right)-$ $g_{\infty}\left(\Lambda_{r}(\lambda) y\right) \mid \leq \bar{k} \lambda^{w_{\max }}$ with $w_{\max }<\nu$ for all $\lambda>1$. Next, $\left|g\left(\Lambda_{r}(\lambda) y\right)-g_{\infty}\left(\Lambda_{r}(\lambda) y\right)\right| \leq \underline{k} \lambda^{w_{\min }}$ for $\lambda \leq 1$ with some $\underline{k}>0$ 
and $w_{\min } \geq 0$ due continuity of $g$ and $g_{\infty}$, additionally $w_{\min } \leq \nu$ (by a proper choice of $\underline{k}$ ), then $k=\max \{\underline{k}, \bar{k}\}$.

Clearly, it is always possible to select the powers in such a way that $0 \leq w_{\min } \leq w_{\max }<\nu$.

Lemma 2. Let a vector field $f: \mathbb{R}^{n} \rightarrow \mathbb{R}^{n}$ be $\left(\mathbf{r},+\infty, f_{\infty}\right)$-homogeneous with degree $\nu, f$ and $f_{\infty}$ be continuous, then for all $x \in \mathbb{R}^{n}$

$$
\left\|f(x)-f_{\infty}(x)\right\| \leq \omega\left(\|x\|_{r}\right), \omega(s)=\left\{\begin{array}{ll}
k s^{w_{\min }} & \text { if } s \leq 1 \\
k s^{w_{\max }} & \text { if } s>1
\end{array},\right.
$$

where $k>0$ and $0 \leq w_{\min } \leq w_{\max }<r_{\max }+\nu$.

Proof: In this case each $f_{i}$ is $\left(\mathbf{r},+\infty, f_{\infty i}\right)$-homogeneous with degree $\nu+r_{i}$ for all $1 \leq i \leq n$, and the result follows Lemma 1.

\section{ROBUSTNESS OF HOMOGENEOUS SYSTEMS}

The ISS property of a $\mathbf{r}$-homogeneous system (1) with degree $\nu>1$ has been investigated in [21], the ISS property of a r-homogeneous system of the form

$$
\dot{x}=f_{0}(x)+G_{0}(x) d
$$

for any admissible degree $\nu \geq-r_{\min }$ (with homogeneous $f_{0}$ and $G_{0}$ ) has been studied in [20]. In this work we would like to propose the conditions of ISS and iISS properties for a $\mathbf{r}$-homogeneous system (1) with any $\nu \geq-r_{\min }$.

Define

$$
\tilde{f}(x, d)=\left[f(x, d)^{T} 0_{m}\right]^{T} \in \mathbb{R}^{n+m},
$$

it is an extended auxiliary vector field for the system (1), where $0_{m}$ is the zero vector of dimension $m$.

Theorem 6. Let the vector field $\tilde{f}$ be homogeneous with the weights $\mathbf{r}=\left[r_{1}, \ldots, r_{n}\right]>0, \tilde{\mathbf{r}}=\left[\tilde{r}_{1}, \ldots, \tilde{r}_{m}\right] \geq 0$ with a degree $\nu \geq-r_{\min }$, i.e. $f\left(\Lambda_{r}(\lambda) x, \Lambda_{\tilde{r}}(\lambda) d\right)=\lambda^{\nu} \Lambda_{r}(\lambda) f(x, d)$ for all $x \in \mathbb{R}^{n}, d \in \mathbb{R}^{m}$ and all $\lambda>0$. Assume that the system (1) is globally asymptotically stable for $d=0$, then the system (1) is

ISS

$$
\text { if } \tilde{r}_{\min }>0 \text {, where } \tilde{r}_{\min }=\min _{1 \leq j \leq m} \tilde{r}_{j}
$$

iISS

$$
\text { if } \tilde{r}_{\min }=0 \text { and } \nu \leq 0 \text {. }
$$

Proof: Under the introduced conditions $f\left(\Lambda_{r}(\lambda) x, 0\right)=\lambda^{\nu} \Lambda_{r}(\lambda) f(x, 0)$ and the system $\dot{x}=f(x, 0)$ is globally asymptotically stable, therefore by Theorem 3 there exists a continuously differentiable, positive definite and radially unbounded Lyapunov function $V: \mathbb{R}^{n} \rightarrow \mathbb{R}_{+}$such that $V\left(\Lambda_{r}(\lambda) x\right)=\lambda^{\mu} V(x)$ for any $\lambda>0$ and $\mu>r_{\max }$ and

$$
D V(y) f(y, 0) \leq-a,\left\|\frac{\partial V(y)}{\partial y}\right\| \leq b \quad \forall y \in S_{r},
$$

where $a>0, b>0$. Note that by definition in this case $\nu+\mu>0$. Due to homogeneity of $\tilde{f}$ and Lipschitz (Hölder) continuity of $f$ with respect to $d$ we have

$$
\begin{gathered}
\|f(y, d)-f(y, 0)\| \leq \sigma(\|d\|) \quad \forall y \in S_{r}, \\
\sigma(s)= \begin{cases}c s^{\varrho_{\min }} & \text { if } s \leq 1 \\
c s^{\varrho_{\max }} & \text { if } s>1\end{cases}
\end{gathered}
$$

for some $c>0$ and $\varrho_{\max } \geq \varrho_{\min }>0$ for the case $\tilde{r}_{\min }>0\left(\varrho_{\max }=\nu+r_{\max }\right.$ is a possible choice). For the case $\tilde{r}_{\min }=0 \mathrm{a}$ relaxed constraint $\varrho_{\min } \geq 0$ has to be satisfied, which follows the continuity of $f$.

Below we will use the coordinate transformation $x=\Lambda_{r}\left(\|x\|_{r}\right) y$, which connects any $x \in \mathbb{R}^{n} \backslash\{0\}$ with the corresponding point $y \in S_{r}$. For the input $d$ we will use the transformation $d=\Lambda_{\tilde{r}}\left(\|x\|_{r}\right) \tilde{d}$, where $\tilde{d} \in \mathbb{R}^{m}$ is a new auxiliary variable, then

$$
\|\tilde{d}\| \leq \rho\left(\|x\|_{r}\right)\|d\|, \rho(s)=\left\{\begin{array}{ll}
s^{-\tilde{r}_{\max }} & \text { if } s \leq 1 \\
s^{-\tilde{r}_{\min }} & \text { if } s>1
\end{array},\right.
$$


where $\tilde{r}_{\max }=\max _{1 \leq j \leq m} \tilde{r}_{j}$. Now let us consider the time derivative of the Lyapunov function $V$ computed for the system (1) for all $x \in \mathbb{R}^{n}$ and $d \in \mathbb{R}^{m}$ :

$$
\begin{aligned}
D V(x) f(x, d) & =\|x\|_{r}^{\nu+\mu} \frac{\partial V(y)}{\partial y} f(y, \tilde{d}) \\
& =\|x\|_{r}^{\nu+\mu} \frac{\partial V(y)}{\partial y} f(y, 0)+\|x\|_{r}^{\nu+\mu} \frac{\partial V(y)}{\partial y}[f(y, \tilde{d})-f(y, 0)] \\
& \leq-a\|x\|_{r}^{\nu+\mu}+b\|x\|_{r}^{\nu+\mu} \sigma(\|\tilde{d}\|) \\
& \leq-a\|x\|_{r}^{\nu+\mu}+b\|x\|_{r}^{\nu+\mu} \gamma\left(\|x\|_{r}\right) \sigma(\|d\|)
\end{aligned}
$$

where

$$
\gamma(s)=\left\{\begin{array}{ll}
s^{-\tilde{r}_{\max } \varrho_{\min }} & \text { if } s \leq 1 \\
s^{-\tilde{r}_{\min } \varrho_{\max }} & \text { if } s>1
\end{array} .\right.
$$

Therefore if $-\tilde{r}_{\min } \varrho_{\max }<0$, which corresponds to $\tilde{r}_{\min }>0$, then $\gamma(s)^{-1} \in \mathcal{K}_{\infty}$ and for $\|d\| \leq \sigma^{-1}\left[a /(2 b) \gamma\left(\|x\|_{r}\right)^{-1}\right]$ we have $D V(x) f(x, d) \leq-0.5 a\|x\|_{r}^{\nu+\mu}$ that implies ISS by Theorem 1 . If $\tilde{r}_{\max } \varrho_{\min }-\mu \leq \nu \leq \tilde{r}_{\min } \varrho_{\max }$, then

$$
\|x\|_{r}^{\nu+\mu} \gamma\left(\|x\|_{r}\right) \leq v\left(\|x\|_{r}\right), \quad v(s)= \begin{cases}1 & \text { if } s \leq 1 \\ s^{\mu} & \text { if } s>1\end{cases}
$$

and for the Lyapunov function $W(x)=\ln [1+V(x)]$ (it is continuously differentiable, positive definite and radially unbounded) we obtain

$$
\begin{aligned}
D W(x) f(x, d) & \leq \frac{-a\|x\|_{r}^{\nu+\mu}+b v\left(\|x\|_{r}\right) \sigma(\|d\|)}{1+V(x)} \\
& \leq-a \frac{\|x\|_{r}^{\nu+\mu}}{1+V(x)}+\tilde{b} \sigma(\|d\|), \tilde{b}>0
\end{aligned}
$$

which implies iISS for (1). Since $\mu>r_{\max }$ can be chosen arbitrary, then we may assume that the inequality $\tilde{r}_{\max } \varrho_{\min }-\mu \leq \nu$ is always satisfied. Since for $\tilde{r}_{\min }>0$ the system is ISS (and iISS by the definition), the only interesting admissible value is $\tilde{r}_{\min }=0$, then $\nu$ has to be non positive.

As we can conclude from this result, for the homogeneous system (1) its robustness (ISS or iISS property) is a function of its degree of homogeneity.

Corollary 1. Let a locally Lipschitz continuous function $f_{0}: \mathbb{R}^{n} \rightarrow \mathbb{R}^{n}$ be $\mathbf{r}$-homogeneous with a degree $\nu$ and asymptotically stable.

If $f(x, d)=f_{0}(x)+d$, i.e. $d$ is an additive disturbance, then the system (1) is ISS for $\nu>-r_{\min }$, and iISS for $\nu=-r_{\min }$.

If $f(x, d)=f_{0}(x+d)$, i.e. $d$ is a measurement noise, then the system (1) is always ISS.

Proof: Take $\tilde{\mathbf{r}}=\mathbf{r}+\nu$ and $\tilde{\mathbf{r}}=\mathbf{r}$ for the additive disturbance and measurement noise cases respectively.

Thus to verify robustness of a homogeneous system with respect to an external input it is enough to establish its asymptotic stability for the case $d=0$ and compute its degree of homogeneity performing some algebraic operations, which is a big advantage of the homogeneity approach, while in the conventional case an ISS/iISS Lyapunov function has to be found [24]. However, the sole homogeneity of $\tilde{f}$ is not enough to claim iISS (ISS), and the case $\tilde{r}_{\min }=0$ with $\nu>0$ is the only exclusion as in the following example for $\tilde{\mathbf{r}}=0$ and $\mathbf{r}=1$ :

$$
\dot{x}=(d-1) x^{\alpha}, \alpha>1
$$

The asymptotically stable system (1) for $d=0$ is finite-time stable if it is homogeneous with a negative degree [25], [26], [27]. Interestingly to note that the finite-time stability and iISS have a similar restriction on the degree of homogeneity (it has to be negative or non positive for iISS), thus the finite-time stability of a homogeneous system implies iISS.

Corollary 2. Let the vector field $\tilde{f}$ be homogeneous with the weights $\mathbf{r}=\left[r_{1}, \ldots, r_{n}\right]>0, \tilde{\mathbf{r}}=\left[\tilde{r}_{1}, \ldots, \tilde{r}_{m}\right] \geq 0$ with a degree $0>\nu \geq-r_{\min }$ and asymptotically stable for $d=0$ (i.e. finite-time stable), then (1) is iISS. 
Theorem 6 also provides a quantitative estimate on the asymptotic gain of (1) in the ISS case.

Corollary 3. Let the system (1) be ISS under conditions of Theorem $6\left(\tilde{r}_{\min }>0\right)$ and $\nu+\mu>\tilde{r}_{\min } \varrho_{\max }$, then its asymptotic gain $\psi$ admits an estimate:

$$
\psi(s) \leq\left\{\begin{array}{ll}
\ell s^{\min \left\{\frac{1}{\tilde{r}_{\max }}, \frac{\varrho_{\min }}{\tilde{r}_{\min } \varrho_{\max }}\right\}} & \text { if } s \leq 1 \\
\ell s^{\max \left\{\frac{\varrho_{\max }}{\tilde{r}_{\max \varrho_{\min }}}, \frac{1}{\tilde{r}_{\min }}\right\}} & \text { if } s>1
\end{array}, \ell>0 .\right.
$$

The case $\nu+\mu \leq \tilde{r}_{\min } \varrho_{\max }$ has no practical importance and will not be treated here.

Proof: Since in the ISS case $\tilde{r}_{\max } \geq \tilde{r}_{\min }>0$ and $\varrho_{\max } \geq \varrho_{\min }>0$ (and $\underline{k}\|x\|_{r}^{\mu} \leq V(x) \leq \bar{k}\|x\|_{r}^{\mu}$ for all $x \in \mathbb{R}^{n}$, where $\underline{k}=\inf _{y \in S_{r}} V(y)$ and $\left.\bar{k}=\sup _{y \in S_{r}} V(y)\right)$, then following the proof of Theorem 6 we obtain:

$$
\begin{aligned}
D V(x) f(x, d) & \leq-a\|x\|_{r}^{\nu+\mu}+b\|x\|_{r}^{\nu+\mu} \gamma\left(\|x\|_{r}\right) \sigma(\|d\|) \\
& \leq-\tilde{a} V(x)^{\frac{\nu+\mu}{\mu}}+\tilde{b} \tilde{\gamma}(V(x)) \sigma(\|d\|)
\end{aligned}
$$

where

$$
\begin{gathered}
\tilde{a}=\bar{k}^{-\frac{\nu+\mu}{\mu}} a, \tilde{b}=\max \left\{\underline{k}^{-\frac{\nu+\mu-\tilde{r}_{\max } \varrho_{\min }}{\mu}}, \underline{k}^{\left.-\frac{\nu+\mu-\tilde{r}_{\min } \varrho_{\max }}{\mu}\right\} b,}\right. \\
\tilde{\gamma}(s)= \begin{cases}s^{\frac{\nu+\mu-\tilde{r}_{\max } \varrho_{\min }}{\mu}} & \text { if } s \leq 1 \\
s^{\frac{\nu+\mu-\tilde{r}_{\min } \varrho_{\max }}{\mu}} & \text { if } s>1\end{cases}
\end{gathered}
$$

Therefore, $D V(x) f(x, d) \leq-0.5 \tilde{a} V(x)^{\frac{\nu+\mu}{\mu}}$ if

$$
\sigma(\|d\|) \leq 0.5 \tilde{a} \tilde{b}^{-1} \begin{cases}V(x)^{\frac{\tilde{r}_{\max } \varrho_{\min }}{\mu}} & \text { if } V(x) \leq 1 \\ V(x)^{\frac{\tilde{r}_{\min } \varrho_{\max }}{\mu}} & \text { if } V(x)>1\end{cases}
$$

and an estimate on the asymptotic gain $\psi$ can be obtained inverting the last inequality:

$$
\begin{aligned}
& \|x\|_{r} \leq \begin{cases}\ell \max \left\{\|d\| \frac{1}{\tilde{r}_{\max }},\|d\| \frac{\varrho_{\min }}{\tilde{r}_{\min } \varrho_{\max }}\right\} & \text { if }\|d\| \leq 1 \\
\ell \max \left\{\|d\| \frac{\varrho_{\max }}{\tilde{r}_{\max \varrho_{\min }}},\|d\| \frac{1}{\tilde{r}_{\min }}\right\} & \text { if }\|d\|>1\end{cases}
\end{aligned}
$$

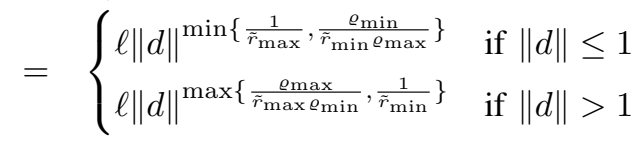

for some $\ell>0$.

The case $\tilde{r}_{\min }=0$ is critical for Theorem 6 , it is possible that the system (1) is ISS while $\tilde{r}_{\min }=0$ as it is shown in the following example:

$$
\begin{aligned}
& \dot{x}_{1}=-x_{1}^{3}+x_{2}^{2} d_{1}, \\
& \dot{x}_{2}=-x_{2}^{7 / 3}+\left|x_{1}\right|^{1 / 2} d_{2},
\end{aligned}
$$

where $\mathbf{r}=\left[\begin{array}{ll}1 & 1.5\end{array}\right], \tilde{\mathbf{r}}=\left[\begin{array}{ll}0 & 3\end{array}\right], \nu=2$ and its ISS Lyapunov function is $V(x)=0.5 x_{1}^{2}+0.5 x_{2}^{2}$.

The conditions of Theorem 6 can be technically relaxed skipping homogeneity of $\tilde{f}$ (homogeneity with respect to $d$ ). It is worth stressing that homogeneity of $\tilde{f}$ is not a restrictive condition since $d$ is an external input, and we can modify dimension or introduce nonlinear change of coordinates for $d$.

Theorem 7. Assume that the system (1) is globally asymptotically stable for $d=0$ and $\mathbf{r}$-homogeneous with a degree $\nu \geq-r_{\min }$, i.e. $f\left(\Lambda_{r}(\lambda) x, 0\right)=\lambda^{\nu} \Lambda_{r}(\lambda) f(x, 0)$ for all $x \in \mathbb{R}^{n}$ and all $\lambda>0$. Let also for all $x \in \mathbb{R}^{n}$ and all $d \in \mathbb{R}^{m}$

$$
\begin{gathered}
\|f(x, d)-f(x, 0)\| \leq \theta\left(\|x\|_{r}\right) \psi(\|d\|)+\phi(\|d\|), \\
\theta(s)=\left\{\begin{array}{ll}
s^{\vartheta_{\min }} & \text { if } s \leq 1 \\
s^{\vartheta_{\max }} & \text { if } s>1
\end{array}, \vartheta_{\max } \geq \vartheta_{\min } \geq 0\right.
\end{gathered}
$$


for some $\psi, \phi \in \mathcal{K}$. Then the system (1) is

ISS

$$
\begin{aligned}
& \text { if } \nu>\vartheta_{\max }-r_{\min } ; \\
& \text { if } \nu=\vartheta_{\max }-r_{\min } \leq 0 .
\end{aligned}
$$

iISS

Proof: Under introduced conditions, by Theorem 3 there exists a continuously differentiable, positive definite and radially unbounded Lyapunov function $V: \mathbb{R}^{n} \rightarrow \mathbb{R}_{+}$such that $V\left(\Lambda_{r}(\lambda) x\right)=\lambda^{\mu} V(x)$ for any $\lambda>0$ and $\mu>r_{\max }$ with $\nu+\mu>0$, and the inequalities (4) are satisfied for $a>0, b>0$. Consider the time derivative of the Lyapunov function $V$ computed for the system (1) for all $x \in \mathbb{R}^{n}$ and $d \in \mathbb{R}^{m}$ using the coordinate transformation $x=\Lambda_{r}\left(\|x\|_{r}\right) y\left(\|x\|_{r}=\left\|\Lambda_{r}\left(\|x\|_{r}\right) y\right\|_{r}\right)$ :

$$
\begin{gathered}
D V(x) f(x, d)=\frac{\partial V(x)}{\partial x} f(x, 0)+\frac{\partial V(x)}{\partial x}[f(x, d)-f(x, 0)] \\
=\|x\|_{r}^{\nu+\mu} \frac{\partial V(y)}{\partial y} f(y, 0)+\|x\|_{r}^{\mu} \frac{\partial V(y)}{\partial y} \Lambda_{r}^{-1}\left(\|x\|_{r}\right)\left[f\left(\Lambda_{r}\left(\|x\|_{r}\right) y, d\right)-f\left(\Lambda_{r}\left(\|x\|_{r}\right) y, 0\right)\right] \\
\leq-a\|x\|_{r}^{\nu+\mu}+b\|x\|_{r}^{\mu}\left\|\Lambda_{r}^{-1}\left(\|x\|_{r}\right)\right\|\left[\theta\left(\left\|\Lambda_{r}\left(\|x\|_{r}\right) y\right\|_{r}\right) \psi(\|d\|)+\phi(\|d\|)\right] \\
\leq-a\|x\|_{r}^{\nu+\mu}+b\|x\|_{r}^{\nu+\mu}\left[\gamma_{1}\left(\|x\|_{r}\right) \psi(\|d\|)+\gamma_{2}\left(\|x\|_{r}\right) \phi(\|d\|)\right],
\end{gathered}
$$

where

$$
\begin{aligned}
& \gamma_{1}(s)= \begin{cases}s^{\vartheta_{\min }-r_{\max }-\nu} & \text { if } s \leq 1 \\
s^{\vartheta_{\max }-r_{\min }-\nu} & \text { if } s>1\end{cases} \\
& \gamma_{2}(s)= \begin{cases}s^{-r_{\max }-\nu} & \text { if } s \leq 1 \\
s^{-r_{\min }-\nu} & \text { if } s>1\end{cases}
\end{aligned}
$$

Therefore if $\vartheta_{\max }-r_{\min }-\nu<0$, then $\gamma_{1}(s)^{-1}$ and $\gamma_{2}(s)^{-1}$ are in $\mathcal{K}_{\infty}$, and for $\|d\| \quad \leq$ $\min \left\{\psi^{-1}\left[a /(4 b) \gamma_{1}\left(\|x\|_{r}\right)^{-1}\right], \phi^{-1}\left[a /(4 b) \gamma_{2}\left(\|x\|_{r}\right)^{-1}\right]\right\}$ we have $D V(x) f(x, d) \leq-0.5 a\|x\|_{r}^{\nu+\mu}$ that, by Theorem 1 , implies the ISS property. If $\vartheta_{\max }-r_{\min }-\nu=0, \nu \leq 0$ and $\vartheta_{\min }-r_{\max }+\mu \geq 0$ (the latter is true since $\mu>r_{\max }$ ), then $\|x\|_{r}^{\nu+\mu} \gamma_{1}\left(\|x\|_{r}\right) \leq v\left(\|x\|_{r}\right)$ and $\|x\|_{r}^{\nu+\mu} \gamma_{2}\left(\|x\|_{r}\right) \leq v\left(\|x\|_{r}\right)$, where $v(s)$ is defined at (6) and for the Lyapunov function $W(x)=\ln [1+V(x)]$ we obtain (7) for $\sigma(s)=2 \max \{\psi(s), \phi(s)\}$, which implies iISS for (1).

In the theorem it is assumed that $\vartheta_{\text {min }} \geq 0$ and $\vartheta_{\max } \in \mathbb{R}$. The condition on $\vartheta_{\min }$ follows continuity of $f$, while the rate of the growth of $f$ at infinity is defined by $\vartheta_{\max }$, and formally there is nor restriction on it except $\vartheta_{\max } \geq \vartheta_{\min }$.

The result of Theorem 7 can be applied for a larger class of systems, which are not necessarily homogeneous (the function $\tilde{f}$ may be non homogeneous). For example, to the system (3) with non homogeneous $G_{0}$ (the result of [20] cannot be used in this case):

$$
\begin{aligned}
& \dot{x}_{1}=-x_{1}+x_{2} d_{1} /\left(1+\left|x_{2}\right|\right), \\
& \dot{x}_{2}=-x_{2}+x_{1}^{1 / 3} d_{2}
\end{aligned}
$$

where $\mathbf{r}=\left[\begin{array}{ll}1 & 1\end{array}\right]$ and $\nu=0$ for $d=0, \vartheta_{\min }=\vartheta_{\max }=1 / 3$.

However, the conditions obtained in Theorem 7 also do not work for the critical case example (8), where $\vartheta_{\text {min }}=0.5$, $\vartheta_{\max }=3$ and the equality $\nu=\vartheta_{\max }-r_{\min }$ is satisfied. A reason of that is hidden in the conservatism of the function $\theta$ computation. Another explanation of this fact is that, in the case $\tilde{r}_{\min }=0$ the system (1) may not admit a $\mathbf{r}$-homogeneous ISS Lyapunov function (both theorems 6 and 7 are based on an ISS Lyapunov function of that type), see also the case of Proposition 1 below, where this hypothesis is proven for the case $\tilde{\mathbf{r}}=0_{m}$.

Proposition 1. Considering $d$ as a constant, let the vector field $f$ be $\mathbf{r}-$ homogeneous with a degree $\nu \geq-r_{\min }$ independent of d, i.e. $f\left(\Lambda_{r}(\lambda) x, d\right)=\lambda^{\nu} \Lambda_{r}(\lambda) f(x, d)$ for all $x \in \mathbb{R}^{n}, d \in \mathbb{R}^{m}$ and all $\lambda>0$. Assume that the system (1) has a $\mathbf{r}-$ homogeneous ISS Lyapunov functions for (1), then (1) is globally asymptotically stable uniformly in $d$.

Proof: Take any $\mathbf{r}$-homogeneous ISS Lyapunov function for the system (1), say $V(x)$ with a degree $\mu>0$ (if $\mu=0$, 
then $V$ is not continuous), then for any $x \in \mathbb{R}^{n}, d \in \mathbb{R}^{m}$ and $y \in S_{r}$ we obtain

$$
D V(x) f(x, d)=\|x\|_{r}^{\nu+\mu} c_{1}(y)+\|x\|_{r}^{\nu+\mu} c_{2}(y, d)
$$

where $c_{1}(y)=\frac{\partial V(y)}{\partial y} f(y, 0)<0$ and $c_{2}(y, d)=\frac{\partial V(y)}{\partial y}[f(y, d)-f(y, 0)]$. Therefore, if $V$ is an ISS Lyapunov function, then for any $\|d\|$ there is $\|x\|_{r}$ such that $D V(x) f(x, d)<0$, which is true if $c_{2}(y, d)<-c_{1}(y)$ only.

However, the case of Proposition 1 still can be useful for the iISS property.

Theorem 8. Let the vector field $f$ be $\mathbf{r}$-homogeneous with a degree $0 \geq \nu \geq-r_{\min }$ considering $d$ as a constant, i.e. $f\left(\Lambda_{r}(\lambda) x, d\right)=\lambda^{\nu} \Lambda_{r}(\lambda) f(x, d)$ for all $x \in \mathbb{R}^{n}, d \in \mathbb{R}^{m}$ and all $\lambda>0$. Then the system (1) is globally asymptotically stable for $d=0$ iff it is iISS.

Proof: First, iISS property implies global asymptotic stability for $d=0$. Second, under introduced conditions $f\left(\Lambda_{r}(\lambda) x, 0\right)=\lambda^{\nu} \Lambda_{r}(\lambda) f(x, 0)$ and the system $\dot{x}=f(x, 0)$ is globally asymptotically stable, therefore by Theorem 3 there exists a continuously differentiable, positive definite and radially unbounded Lyapunov function $V: \mathbb{R}^{n} \rightarrow \mathbb{R}_{+}$such that $V\left(\Lambda_{r}(\lambda) x\right)=\lambda^{\mu} V(x)$ for any $\lambda>0$ and $\mu>r_{\max }(\nu+\mu>0)$, and the inequalities (4) are satisfied for $a>0, b>0$. Due to continuity of $f$ with respect to $d$ we have for some $\sigma \in \mathcal{K}$

$$
\|f(y, d)-f(y, 0)\| \leq \sigma(\|d\|) \quad \forall y \in S_{r} .
$$

Now let us consider the time derivative of the Lyapunov function $V$ computed for the system (1) for all $x \in \mathbb{R}^{n}$ and $d \in \mathbb{R}^{m}$ using the coordinate transformation $x=\Lambda_{r}\left(\|x\|_{r}\right) y$ :

$$
\begin{aligned}
D V(x) f(x, d) & =\|x\|_{r}^{\nu+\mu} \frac{\partial V(y)}{\partial y} f(y, 0)+\|x\|_{r}^{\nu+\mu} \frac{\partial V(y)}{\partial y}[f(y, d)-f(y, 0)] \\
& \leq-a\|x\|_{r}^{\nu+\mu}+b\|x\|_{r}^{\nu+\mu} \sigma(\|d\|)
\end{aligned}
$$

Therefore, if $\nu \leq 0$, then $\|x\|_{r}^{\nu+\mu} \leq v\left(\|x\|_{r}\right)$, where $v(s)$ is defined in (6), and for the Lyapunov function $W(x)=\ln [1+V(x)]$ we obtain (7) that according to Theorem 1 implies iISS for (1).

This result can be applied, for example, to "bilinear" systems:

$$
\dot{x}=f_{0}(x)+\sum_{i=1}^{m} f_{i}\left(x d_{i}\right),
$$

where all $f_{i}, i=\overline{0, m}$ are $\mathbf{r}$-homogeneous functions of the same degree with respect to $x, f_{i}(0)=0$ (the simplest example is $f_{i}(x)=A_{i} x$, where $A_{i} \in \mathbb{R}^{n \times n}$ ). According to Theorem 8 , if in (9) the system $\dot{x}=f_{0}(x)$ is asymptotically stable and the homogeneity degree is non-positive, then the system is iISS.

To finish comparison of theorems 6 and 7 note that the conditions of Theorem 7 may be more restrictive than in Theorem 6 , as it can be demonstrated in the following example:

$$
\begin{aligned}
& \dot{x}_{1}=-x_{1}^{3}+x_{2}^{1 / 3} d_{1}, \\
& \dot{x}_{2}=-x_{2}^{5 / 3}+x_{1}^{3} d_{2},
\end{aligned}
$$

where $\mathbf{r}=\left[\begin{array}{ll}1 & 3\end{array}\right], \tilde{\mathbf{r}}=\left[\begin{array}{ll}2 & 2\end{array}\right], \nu=2$ and it is ISS by Theorem 6 (it also has a homogeneous ISS Lyapunov function $V(x)=x_{1}^{6} / 6+x_{2}^{2} / 2$ ), but Theorem 7 does not work since $\vartheta_{\min }=1, \vartheta_{\max }=3$ and $\nu=\vartheta_{\max }-r_{\min }$. In addition, the iISS condition in Theorem 7 implicitly needs $\vartheta_{\max }<r_{\min }$. Another interpretation of the ISS condition of Theorem 7 is that the system (1) has local approximation at infinity $f(x, 0)$.

Finally consider an example, for which a strict Lyapunov function is not known, but using theorems 6 or 7 it is possible to establish ISS property. Let us consider a planar nonlinear system:

$$
\begin{aligned}
& \dot{e}_{1}=e_{2}-l_{1}\left\lfloor e_{1}+d_{1}\right\rceil^{\beta}, \\
& \dot{e}_{2}=-l_{2}\left\lfloor e_{1}+d_{1}\right\rceil^{2 \beta-1}+d_{2},
\end{aligned}
$$


where $e_{1} \in \mathbb{R}, e_{2} \in \mathbb{R}$ are the states, $d_{1} \in \mathbb{R}, d_{2} \in \mathbb{R}$ are external inputs, $l_{1}>0, l_{2}>0$ and $\beta \in\left(\frac{1}{2}, 1\right)$ are the parameters. Such a system describes dynamics of estimation error when analyzing a finite-time observer/differentiator [28]. In this case $d_{1}$ represents the measurement noise and $d_{2}$ models an external disturbance or model mismatch. The system (10) is homogeneous for $\mathbf{r}=\left[\begin{array}{ll}1 & \beta\end{array}\right]$ and $\tilde{\mathbf{r}}=\left[\begin{array}{lll}1 & 2 \beta-1\end{array}\right]$ with degree $\nu=\beta-1\left(\nu \geq-r_{\min }\right)$. To show that for $d_{1}=d_{2}=0$ the system is asymptotically stable we can consider a Lyapunov function $V\left(e_{1}, e_{2}\right)=0.5 l_{2} \beta^{-1} e_{1}^{2 \beta}+0.5 e_{2}^{2}$, which derivative for (10) takes the form $\dot{V}=l_{2} e_{1}^{2 \beta-1}\left(e_{2}-l_{1}\left\lfloor e_{1}\right\rceil^{\beta}\right)-l_{2} e_{2}\left\lfloor e_{1}\right\rceil^{2 \beta-1}=-l_{1} l_{2}\left|e_{1}\right|^{3 \beta-1}$, i.e. $V$ is not a strict Lyapunov function for the system (10). Therefore, the system is Lyapunov stable $(\dot{V} \leq 0)$ and all its trajectories are attracted by the origin (the origin is the only invariant solution on the line $e_{1}=0$ ), thus the system (10) is globally asymptotically stable. In fact, since it is homogeneous with a negative degree it is finite-time stable [29]. By Theorem 6 , since $\tilde{r}_{\min }>0$, the system is ISS with respect to $d_{1}$ and $d_{2}$. In [28] there is also another example, for which a strict Lyapunov function is not known, but using homogeneity it is possible to prove ISS property, it is a nonlinear homogeneous controller from [30].

\section{ROBUSTNESS OF LOCALLY HOMOGENEOUS SYSTEMS}

The ISS property of locally homogeneous systems has been analyzed in [18], it was shown there that if the system (1) is locally homogeneous at 0 and $+\infty$, and all approximations and the system itself are globally asymptotically stable for $d=0$, then (1) is ISS. First we are going to propose a variant of that proof for approximation at infinity and, next, we will extend it for the systems not homogeneous with respect to $d$.

Now assume that the system is locally homogeneous at infinity. Define

$$
\tilde{f}_{\infty}(x, d)=\left[f_{\infty}^{T}(x, d) 0_{m}^{T}\right]^{T}
$$

Assumption 1. Let the vector field $\tilde{f}$ be $\left((\mathbf{r}, \tilde{\mathbf{r}}),+\infty, \tilde{f}_{\infty}\right)$-homogeneous with the weights $\mathbf{r}=\left[r_{1}, \ldots, r_{n}\right]>$ $0, \tilde{\mathbf{r}}=\left[\tilde{r}_{1}, \ldots, \tilde{r}_{m}\right]>0$ and degree $\nu>-r_{\min }$, i.e. for any $\epsilon>0$ there is a $\lambda_{\epsilon}>0$ such that $\sup _{\lambda \geq \lambda_{\epsilon}}\left\|\lambda^{-\nu} \Lambda_{r}^{-1}(\lambda) f\left(\Lambda_{r}(\lambda) y, \Lambda_{\tilde{r}}(\lambda) d\right)-f_{\infty}(y, d)\right\| \leq \epsilon$ for all $y \in S_{r}$ and all $d \in S_{\tilde{r}}$, where $f_{\infty}$ is a locally Lipschitz continuous function.

Since $\left\|\tilde{f}(x, d)-\tilde{f}_{\infty}(x, d)\right\|=\left\|f(x, d)-f_{\infty}(x, d)\right\|$, define $g(x, d)=f(x, d)-f_{\infty}(x, d)$, then by Lemma 1 in this case for all $x \in \mathbb{R}^{n}$ and $d \in \mathbb{R}^{m}$ we have

$$
\begin{aligned}
\left|g_{i}(x, d)\right| & \leq \omega_{i}\left(\|x\|_{r}+\|d\|_{\tilde{r}}\right), \\
\omega_{i}(s) & = \begin{cases}k s^{w_{\min }^{i}} & \text { if } s \leq 1 \\
k s^{w_{\max }^{i}} & \text { if } s>1\end{cases}
\end{aligned}
$$

for all $i=\overline{1, n}$, where $k>0, w_{\min }^{i} \geq 0$ and $w_{\max }^{i}=r_{i}+\nu-\delta<r_{i}+\nu$ for some $\delta>0$.

Theorem 9. Let Assumption 1 be satisfied. Assume that the system $\dot{x}=f_{\infty}(x, 0)$ is globally asymptotically stable, then the system (1) is ISpS.

Proof: Under the introduced conditions the system $\dot{x}=f_{\infty}(x, d)$ is homogeneous with the weights $(\mathbf{r}, \tilde{\mathbf{r}})$ and the degree $\nu$, and it is globally asymptotically stable for $d=0$, then by Theorem 3 there exists a continuously differentiable, positive definite and radially unbounded Lyapunov function $V: \mathbb{R}^{n} \rightarrow \mathbb{R}_{+}$such that $V\left(\Lambda_{r}(\lambda) x\right)=\lambda^{\mu} V(x)$ for any $\lambda>0$ and $\mu>r_{\max }($ with $\nu+\mu>0)$, and

$$
D V(y) f_{\infty}(y, 0) \leq-a,\left\|\frac{\partial V(y)}{\partial y}\right\| \leq b \quad \forall y \in S_{r}
$$

where $a>0, b>0$. We will use the coordinate transformation $x=\Lambda_{r}\left(\|x\|_{r}\right) y$ and $d=\Lambda_{\tilde{r}}\left(\|x\|_{r}\right) \tilde{d}$, where $\tilde{d} \in \mathbb{R}^{m}$, then the 
inequality (5) is satisfied. Due to homogeneity and continuity of $f_{\infty}$ with respect to $d$ we have

$$
\begin{gathered}
\left\|f_{\infty}(y, d)-f_{\infty}(y, 0)\right\| \leq \sigma(\|d\|) \quad \forall y \in S_{r}, \\
\sigma(s)= \begin{cases}c s^{\varrho_{\min }} & \text { if } s \leq 1 \\
c s^{\varrho_{\max }} & \text { if } s>1\end{cases}
\end{gathered}
$$

for some $c>0$ and $\varrho_{\max } \geq \varrho_{\min }>0$.

Now let us consider the time derivative of the Lyapunov function $V$ computed for the system (1) for all $x \in \mathbb{R}^{n}$ and $d \in \mathbb{R}^{m}$ :

$$
\begin{aligned}
& D V(x) f(x, d)=D V(x) f_{\infty}(x, d)+D V(x)\left[f(x, d)-f_{\infty}(x, d)\right] \\
= & \|x\|_{r}^{\nu+\mu} \frac{\partial V(y)}{\partial y} f_{\infty}(y, \tilde{d})+\|x\|_{r}^{\mu} \frac{\partial V(y)}{\partial y} \Lambda_{r}^{-1}\left(\|x\|_{r}\right)\left[f(x, d)-f_{\infty}(x, d)\right] \\
= & \|x\|_{r}^{\nu+\mu} \frac{\partial V(y)}{\partial y} f_{\infty}(y, 0)+\|x\|_{r}^{\nu+\mu} \frac{\partial V(y)}{\partial y}\left[f_{\infty}(y, \tilde{d})-f_{\infty}(y, 0)\right] \\
& +\|x\|_{r}^{\mu} \frac{\partial V(y)}{\partial y} \Lambda_{r}^{-1}\left(\|x\|_{r}\right)\left[f(x, d)-f_{\infty}(x, d)\right] .
\end{aligned}
$$

Define $s(x, d)=\Lambda_{r}^{-1}\left(\|x\|_{r}\right)\left[f(x, d)-f_{\infty}(x, d)\right]$, we have

$$
\begin{aligned}
\|x\|_{r}^{\nu+\mu} \frac{\partial V(y)}{\partial y} f_{\infty}(y, 0) & \leq-a\|x\|_{r}^{\nu+\mu}, \\
\|s(x, d)\| & \leq \sqrt{n} \max _{1 \leq i \leq n}\left|s_{i}(x, d)\right| \\
& \leq \tau\left(\|x\|_{r}+\|d\|_{\tilde{r}}\right), \\
\|x\|_{r}^{\mu} \frac{\partial V(y)}{\partial y}\left[f_{\infty}(y, \tilde{d})-f_{\infty}(y, 0)\right] & \leq b\|x\|_{r}^{\nu+\mu} \sigma(\|\tilde{d}\|) \\
& \leq b \gamma\left(\|x\|_{r}\right) \sigma(\|d\|),
\end{aligned}
$$

where

$$
\begin{gathered}
\gamma(s)=\left\{\begin{array}{ll}
s^{\nu+\mu-\tilde{r}_{\max } \varrho_{\min }} & \text { if } s \leq 1 \\
s^{\nu+\mu-\tilde{r}_{\min } \varrho_{\max }} & \text { if } s>1
\end{array},\right. \\
\tau(s)=\left\{\begin{array}{ll}
\sqrt{n} k s^{w_{\min }-r_{\max }} & \text { if } s \leq 1 \\
\sqrt{n} k s^{w_{\max }} & \text { if } s>1
\end{array},\right. \\
w_{\min }=\min _{1 \leq i \leq n} w_{\min }^{i}, w_{\max }=\max _{1 \leq i \leq n} w_{\max }^{i}-r_{i} \leq \nu-\delta
\end{gathered}
$$

and the function $\rho$ from (5) has been used to calculate $\gamma$. From these inequalities we obtain

$$
\begin{aligned}
D V(x) f(x, d) \leq & -a\|x\|_{r}^{\nu+\mu}+b \gamma\left(\|x\|_{r}\right) \sigma(\|d\|) \\
& +b\|x\|_{r}^{\mu}\left[\tau\left(2\|x\|_{r}\right)+\tau\left(2\|d\|_{\tilde{r}}\right)\right] .
\end{aligned}
$$

By the homogeneous norm definition there exists a class $\mathcal{K}_{\infty}$ function $\chi$ such that $\tau\left(2\|d\|_{\tilde{r}}\right) \leq \chi(\|d\|)$ for all $d \in \mathbb{R}^{m}$, then

$$
\begin{aligned}
D V(x) f(x, d) \leq & -a\|x\|_{r}^{\nu+\mu}+z \kappa\left(\|x\|_{r}\right) \\
& +\left[\gamma\left(\|x\|_{r}\right)+\|x\|_{r}^{\mu}\right] \tilde{\sigma}(\|d\|)
\end{aligned}
$$

where $z=2^{w_{\max }} \sqrt{n} k b, \tilde{\sigma}(s)=b \max \{\sigma(s), \chi(s)\}$ and

$$
\kappa(s)=\left\{\begin{array}{ll}
s^{\mu+w_{\min }-r_{\max }} & \text { if } s \leq 1 \\
s^{\mu+\nu-\delta} & \text { if } s>1
\end{array} .\right.
$$


The power of $\kappa$ for the case $s>1$ is less than $\nu+\mu$ and $\mu+w_{\min }-r_{\max } \geq 0$, then $\kappa\left(\|x\|_{r}\right) \leq 1+\|x\|_{r}^{\mu+\nu-\delta}$ and we get

$$
\begin{aligned}
-a\|x\|_{r}^{\nu+\mu}+z \kappa\left(\|x\|_{r}\right) & \leq-a\|x\|_{r}^{\nu+\mu}+z+z\|x\|_{r}^{\mu+\nu-\delta} \\
& \leq-0.5 a\|x\|_{r}^{\nu+\mu}+c
\end{aligned}
$$

where $c=z+0.5 a[1-\epsilon]^{-1}\left[2 z a^{-1}\left(1-\epsilon^{-1}\right)\right]^{\epsilon}$ and $\epsilon=(\nu+\mu) / \delta$ (i.e. $-0.5 a s^{\nu+\mu}+z s^{\nu+\mu-\delta} \leq 0.5 a[1-\epsilon]^{-1}\left[2 z\left(1-\epsilon^{-1}\right)\right]^{\epsilon}$

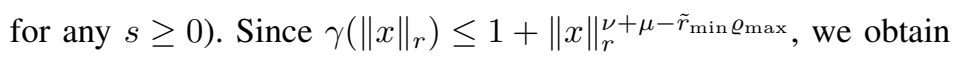

$$
D V(x) f(x, d) \leq-0.5 a\|x\|_{r}^{\nu+\mu}+c+\left[1+\|x\|_{r}^{\nu+\mu-\tilde{r}_{\min } \varrho_{\max }}+\|x\|_{r}^{\mu}\right] \tilde{\sigma}(\|d\|) .
$$

Since $\tilde{r}_{\min }>0$ and $-\tilde{r}_{\min } \varrho_{\max }<0$, then from Young's inequality $\left(s v \leq \frac{s^{p}}{p}+\left(1-\frac{1}{p}\right) v^{\frac{p}{p-1}}\right.$ for any $s, v \in \mathbb{R}_{+}$and $\left.p \geq 1\right)$ we have:

$$
\begin{aligned}
s^{\nu+\mu-\tilde{r}_{\min } \varrho_{\max } v} & \leq \frac{a}{8} s^{\nu+\mu}+\pi_{1}^{-1} v^{\pi_{1}} \phi_{1} \\
s^{\mu} v & \leq \frac{a}{8} s^{\nu+\mu}+\pi_{2}^{-1} v^{\pi_{2}} \phi_{2}
\end{aligned}
$$

where $\pi_{1}=\frac{\nu+\mu}{\tilde{r}_{\min } \varrho_{\max }}, \pi_{2}=\frac{\nu+\mu}{\nu}$ and $\phi_{i}=\left[\frac{a}{8} \frac{\pi_{i}}{\pi_{i}-1}\right]^{1-\pi_{i}}$ for $i=1,2$. Consequently,

$$
D V(x) f(x, d) \leq-0.25 a\|x\|_{r}^{\nu+\mu}+\theta(\|d\|)+c,
$$

where $\theta(s)=\tilde{\sigma}(s)+\sum_{i=1}^{2} \pi_{i}^{-1} \tilde{\sigma}(s)^{\pi_{i}} \phi_{i}$, which implies ISpS property by Theorem 1 .

For an example, consider the system:

$$
\begin{aligned}
& \dot{x}_{1}=x_{1}-x_{1}^{3}+x_{2}\left|x_{1}\right|^{0.75} d \\
& \dot{x}_{2}=x_{2}-\left|x_{2}\right| x_{2}+\left|x_{1}\right|^{3.5}\left|x_{2}\right|^{0.125} d,
\end{aligned}
$$

which is $\left((\mathbf{r}, \tilde{\mathbf{r}}),+\infty, \tilde{f}_{\infty}\right)$-homogeneous with the weights $\mathbf{r}=[1,2], \tilde{\mathbf{r}}=0.25$ and degree $\nu=2$ with $f_{\infty}(x, d)=\left[-x_{1}^{3}+\right.$ $\left.x_{2}\left|x_{1}\right|^{0.75} d-\left|x_{2}\right| x_{2}+\left|x_{1}\right|^{3.5}\left|x_{2}\right|^{0.125} d\right]^{T}$. The linearization of the system is unstable and it is hard to simulate this system in order to check its stability since it is very stiff. However, since all conditions of Theorem 9 are satisfied, the system is ISpS.

Corollary 4. Let all conditions of Theorem 9 be satisfied, $a>2^{w_{\max }} \sqrt{n} k b$ and $w_{\min }-r_{\max } \geq \nu$, then the system (1) is ISS.

Proof: As we can conclude from (12), under these additional (restrictive) conditions there is some $\varepsilon>0$ such that

$$
D V(x) f(x, d) \leq-\varepsilon\|x\|_{r}^{\nu+\mu}+\left[\gamma\left(\|x\|_{r}\right)+\|x\|_{r}^{\mu}\right] \tilde{\sigma}(\|d\|)
$$

since the power of the functions $\gamma$ is less than $\nu+\mu\left(\tilde{r}_{\min }>0\right)$, then there is a function $\varsigma \in \mathcal{K}$ such that for $\|d\| \leq \varsigma\left(\|x\|_{r}\right)$ we have

$$
D V(x) f(x, d) \leq-0.5 \varepsilon\|x\|_{r}^{\nu+\mu}
$$

Corollary 5. Let a locally Lipschitz continuous vector field $f_{0}: \mathbb{R}^{n} \rightarrow \mathbb{R}^{n}$ be $\left(\mathbf{r},+\infty, f_{\infty}\right)$-homogeneous with a degree $\nu>-r_{\min }$ and the system $\dot{x}=f_{\infty}(x)$ be asymptotically stable. If $f(x, d)=f_{0}(x)+d$, i.e. $d$ is an additive disturbance, or $f(x, d)=f_{0}(x+d)$, i.e. $d$ is a measurement noise, then the system (1) is ISpS.

Proof: Take $\tilde{\mathbf{r}}=\mathbf{r}+\nu$ for the additive disturbance case, and $\tilde{\mathbf{r}}=\mathbf{r}$ for the measurement noise. The result follows Theorem 9.

There is a modification of Theorem 9, which skips homogeneity with respect to $d$ in Assumption 1 .

Assumption 2. Let the vector field $f$ be $\left(\mathbf{r},+\infty, f_{\infty}\right)$-homogeneous with degree $\nu>-r_{\min }$ for $d=0$, i.e. for any $\epsilon>0$ there is a $\lambda_{\epsilon}>0$ such that $\sup _{\lambda \geq \lambda_{\epsilon}}\left\|\lambda^{-\nu} \Lambda_{r}^{-1}(\lambda) f\left(\Lambda_{r}(\lambda) y, 0\right)-f_{\infty}(y, 0)\right\| \leq \epsilon$ for all $y \in S_{r}$, where $f_{\infty}$ is a locally Lipschitz 
continuous function. Let also for all $x \in \mathbb{R}^{n}$ and all $d \in \mathbb{R}^{m}$ the inequality

$$
\begin{gathered}
\|f(x, d)-f(x, 0)\| \leq \theta\left(\|x\|_{r}\right) \psi(\|d\|)+\phi(\|d\|), \\
\theta(s)=\left\{\begin{array}{ll}
s^{\vartheta_{\min }} & \text { if } s \leq 1 \\
s^{\vartheta_{\max }} & \text { if } s>1
\end{array}, \vartheta_{\max } \geq \vartheta_{\min } \geq 0\right.
\end{gathered}
$$

be satisfied for some $\psi, \phi \in \mathcal{K}$ and $\nu>\vartheta_{\max }-r_{\min }$.

Define $g(x)=f(x, 0)-f_{\infty}(x, 0)$, by Lemma 1 in this case for all $x \in \mathbb{R}^{n}$ we have

$$
\begin{aligned}
\left|g_{i}(x)\right| & \leq \omega_{i}\left(\|x\|_{r}\right), \\
\omega_{i}(s) & =\left\{\begin{array}{ll}
k s^{w_{\min }^{i}} & \text { if } s \leq 1 \\
k s^{w_{\max }^{i}} & \text { if } s>1
\end{array},\right.
\end{aligned}
$$

where $k>0, w_{\min }^{i} \geq 0$ and $w_{\max }^{i}=r_{i}+\nu-\delta<r_{i}+\nu$ for some $\delta>0$.

Theorem 10. Let Assumption 2 be satisfied. Assume that the system $\dot{x}=f_{\infty}(x, 0)$ is globally asymptotically stable, then the system (1) is ISpS.

Proof: Under the introduced conditions the system $\dot{x}=f_{\infty}(x, 0)$ is $\mathbf{r}$-homogeneous with the degree $\nu$, and it is globally asymptotically stable for $d=0$, then by Theorem 3 there exists a continuously differentiable, positive definite and radially unbounded Lyapunov function $V: \mathbb{R}^{n} \rightarrow \mathbb{R}_{+}$such that $V\left(\Lambda_{r}(\lambda) x\right)=\lambda^{\mu} V(x)$ for any $\lambda>0$ and $\mu>r_{\max }$ (with $\nu+\mu>0$ ), and (11) holds for some $a>0, b>0$. Consider the time derivative of $V$ computed for (1) for all $x \in \mathbb{R}^{n}$ and $d \in \mathbb{R}^{m}$ (using the coordinate transformation $\left.x=\Lambda_{r}\left(\|x\|_{r}\right) y\right)$ :

$$
\begin{aligned}
& D V(x) f(x, d)= D V(x) f(x, 0)+D V(x)[f(x, d)-f(x, 0)] \\
&= D V(x) f_{\infty}(x, 0)+D V(x)[f(x, 0) \\
&\left.-f_{\infty}(x, 0)\right]+D V(x)[f(x, d)-f(x, 0)] \\
& \leq \quad-a\|x\|_{r}^{\nu+\mu}+\|x\|_{r}^{\mu} \frac{\partial V(y)}{\partial y} \Lambda_{r}^{-1}\left(\|x\|_{r}\right)[f(x, d) \\
&\quad-f(x, 0)]+\|x\|_{r}^{\mu} \frac{\partial V(y)}{\partial y} s(x) \\
& \leq \quad-a\|x\|_{r}^{\nu+\mu}+\kappa\left(\|x\|_{r}\right)+\gamma\left(\|x\|_{r}\right) \psi\left(\|d\|^{\prime}\right) \\
&+\tau\left(\|x\|_{r}\right) \phi\left(\|d\|^{\prime},\right.
\end{aligned}
$$

where $s(x)=\Lambda_{r}^{-1}\left(\|x\|_{r}\right) g(x), \gamma(s)=\tau \circ \theta(s)$ and

$$
\begin{aligned}
\tau(s) & = \begin{cases}s^{\mu-r_{\max }} & \text { if } s \leq 1 \\
s^{\mu-r_{\min }} & \text { if } s>1\end{cases} \\
\kappa(s) & =\sqrt{n} b k s^{\mu} \max _{1 \leq i \leq n}\left|s_{i}(x)\right| \\
& = \begin{cases}s^{\mu+w_{\min }-r_{\max }} & \text { if } s \leq 1 \\
s^{\mu+w_{\max }} & \text { if } s>1\end{cases}
\end{aligned}
$$

$w_{\min }=\min _{1 \leq i \leq n} w_{\min }^{i}$ and $w_{\max }=\max _{1 \leq i \leq n} w_{\max }^{i}-r_{i} \leq \nu-\delta$. The powers of $\gamma, \kappa$ and $\tau$ for the case $s>1$ are less than $\nu+\mu$ (since $\mu+\vartheta_{\max }-r_{\min }<\mu+\nu$ and $\mu-r_{\min }<\mu+\nu$ for $\nu>\max \left\{\vartheta_{\max }-r_{\min },-r_{\min }\right\}, \mu+w_{\max } \leq \mu+\nu-\delta<\mu+\nu$ ), then the negative term with the power $\nu+\mu$ would dominate $\gamma, \kappa$ and $\tau$ for sufficiently high values of $\|x\|_{r}$. Next the proof repeats the technical steps of the proof of Theorem 9 .

Theorems 9 and 10 extend the conditions of theorems 6 and 7 on the case of local homogeneity at infinity. However, in the local case the difference between applicability conditions of theorems 9 and 10 is minor, the main advantage is that the local 
approximation at infinity may be failed to exist for both variables $x$ and $d$ (the case of Theorem 9), but it may exist for $d=0$ and Theorem 10 can be applied int this case.

Consider the following example

$$
\begin{aligned}
& \dot{x}_{1}=-x_{1}+\frac{\left|x_{1}\right|^{1 / 6} d}{1+\left|x_{2}\right|^{2}}+x_{2}^{2}, \\
& \dot{x}_{2}=-x_{2}+\left|x_{1}\right|^{1 / 6} d .
\end{aligned}
$$

For $d \neq 0$ this system has no homogeneous approximation at infinity since the term $\frac{\left|x_{1}\right|^{1 / 6} d}{1+\left|x_{2}\right|^{2}}$ has no uniform bound on $S_{(r, \tilde{r})}$ for any $\mathbf{r}$ and $\tilde{\mathbf{r}}$, thus Theorem 9 or result of [18] cannot be applied here. For $d=0$ and $\mathbf{r}=[20.5]$ the system (13) is homogeneous at infinity with

$$
f_{\infty}(x)=\left[\begin{array}{l}
-x_{1} \\
-x_{2}
\end{array}\right]
$$

for degree $\nu=0$, which is clearly asymptotically stable. A direct calculation shows that $\vartheta_{\min }=\vartheta_{\max }=1 / 3$. Therefore, since $\nu>\vartheta_{\max }-r_{\min }$, Assumption 2 is satisfied and according to Theorem 10 the system (13) is ISpS.

\section{AN EXTENSION TO IOSS AND OSS PROPERTIES}

Now consider the system (1) with the output (2). We always can represent this system in the form

$$
\dot{x}=f(x, d)=F(x, y, d)
$$

for a suitably defined $F: \mathbb{R}^{n+m+p} \rightarrow \mathbb{R}^{n}$. Clearly, if the ISS conditions of theorems 6 and 7 are satisfied for (14) with an auxiliary input $(y, d)$, then the system (1), (2) is IOSS (OSS for $d=0$ ). The formulations of theorems 6 and 7 for this case are given below (the proofs are skipped for brevity due to their simplicity).

Theorem 11. Let the vector field $\tilde{F}(x, y, d)=\left[F(x, y, d)^{T} 0_{m+p}\right]^{T} \in \mathbb{R}^{n+m+p}$ be homogeneous with the weights $\mathbf{r}=$ $\left[r_{1}, \ldots, r_{n}\right]>0, \hat{\mathbf{r}}=\left[\hat{r}_{1}, \ldots, \hat{r}_{p}\right]>0, \tilde{\mathbf{r}}=\left[\tilde{r}_{1}, \ldots, \tilde{r}_{m}\right]>0$ with a degree $\nu \geq-r_{\min }$, i.e. $F\left(\Lambda_{r} x, \Lambda_{\hat{r}} y, \Lambda_{\tilde{r}} d\right)=\lambda^{\nu} \Lambda_{r} F(x, y, d)$ for all $x \in \mathbb{R}^{n}, y \in \mathbb{R}^{p}, d \in \mathbb{R}^{m}$ and all $\lambda>0$. Assume that the system (14) is globally asymptotically stable for $y=d=0$, then the system (1), (2) is IOSS (OSS for the case $d=0$ ).

Theorem 12. Assume that the system (14) is globally asymptotically stable for $y=d=0$ and $\mathbf{r}$-homogeneous with a degree $\nu \geq-r_{\min }$, i.e. $F\left(\Lambda_{r} x, 0,0\right)=\lambda^{\nu} \Lambda_{r} F(x, 0,0)$ for all $x \in \mathbb{R}^{n}$ and all $\lambda>0$. Let also for all $x \in \mathbb{R}^{n}, y \in \mathbb{R}^{p}$ and all $d \in \mathbb{R}^{m}$

$$
\begin{gathered}
\|F(x, y, d)-F(x, y, 0)\| \leq \theta\left(\|x\|_{r}\right) \psi(\|(y, d)\|)+\phi(\|(y, d)\|), \\
\theta(s)=\left\{\begin{array}{ll}
s^{\vartheta_{\min }} & \text { if } s \leq 1 \\
s^{\vartheta_{\max }} & \text { if } s>1
\end{array}, \vartheta_{\min } \geq 0, \vartheta_{\max } \in \mathbb{R}\right.
\end{gathered}
$$

for some $\psi, \phi \in \mathcal{K}$ and $\nu>\vartheta_{\max }-r_{\min }$. Then the system (1), (2) is IOSS (OSS for the case $d=0$ ).

The integral versions of IOSS and OSS properties can be obtained using the iISS conditions of theorems 6 and 7.

For an illustration consider

$$
f(x, d)=\left[\begin{array}{c}
-x_{1}^{3}+x_{1} x_{3}^{2}+x_{2} d_{1} \\
-x_{2}^{5}-x_{3}^{2} d_{2} \\
x_{3}-x_{1}^{2} x_{3}+x_{2}^{4} d_{3}
\end{array}\right],
$$

where for $d=0$ the system (1) is not homogeneous and unstable due to the third equation. Now define $y=x_{3}$, then the system (14) with the vector field

$$
F(x, y, d)=\left[\begin{array}{c}
-x_{1}^{3}+x_{1} x_{3}^{2}+x_{2} d_{1} \\
-x_{2}^{5}-x_{3}^{2} d_{2} \\
2 y-x_{3}-x_{1}^{2} x_{3}+x_{2}^{4} d_{3}
\end{array}\right]
$$

is homogeneous with the weights $\mathbf{r}=\left[\begin{array}{lll}1 & 0.5 & 1\end{array}\right], \hat{\mathbf{r}}=3$ and $\tilde{\mathbf{r}}=\left[\begin{array}{lll}2.5 & 0.5 & 1\end{array}\right]$ for $\nu=2$ and the system (1), (2) is IOSS since for $y=d=0$ it admits the Lyapunov function $V(x)=0.5\left[x_{1}^{2}+x_{2}^{2}+x_{3}^{2}\right]$ (all conditions of Theorem 11 are satisfied). 


\section{CONCLUSION}

Several conditions of the ISS property and related notions (iISS, IOSS, OSS) have been developed based on the homogeneity theory. The advantage of these conditions is that the system robustness can be checked after its asymptotic stability in the unperturbed case provided that some algebraic homogeneity constraints are satisfied for the system equations (globally or locally). All results are obtained for generic nonlinear systems. Several examples are proposed showing efficiency of the proposed theory and its limitations.

\section{REFERENCES}

[1] J. Doyle, B. Francis, and A. Tannenbaum, Feedback Control Systems. Mac Millan Publishing Co, 1992.

[2] D. J. Hill and P. J. Moylan, "Dissipative dynamical systems: basic input-output and state properties," J. Franklin Inst., vol. 309, no. 5, pp. 327-357, 1980.

[3] A. van der Schaft, $L_{2}$-gain and passivity techniques in nonlinear control, vol. 218 of Lecture Notes in Control and Information Sciences. London: Springer-Verlag London Ltd., 1996.

[4] E. D. Sontag, "The ISS philosophy as a unifying framework for stability-like behavior," in Nonlinear control in the year 2000 , Vol. 2 (Paris), vol. 259 of Lecture Notes in Control and Inform. Sci., pp. 443-467, London: Springer, 2001.

[5] M. Vidyasagar, Input-output analysis of large-scale interconnected systems, vol. 29 of Lecture Notes in Control and Information Sciences. Berlin: Springer-Verlag, 1981. Decomposition, well-posedness and stability.

[6] J. C. Willems, "Dissipative dynamical systems. I. General theory," Arch. Rational Mech. Anal., vol. 45, pp. 321-351, 1972.

[7] E. D. Sontag, "Smooth stabilization implies coprime factorization," IEEE Trans. Automat. Control, vol. 34, no. 4, pp. 435-443, 1989.

[8] S. Dashkovskiy, D. Efimov, and E. Sontag, "Input to state stability and allied system properties," Automation and Remote Control, vol. 72, no. 8, pp. 1579-1614, 2011.

[9] V. Zubov, "On systems of ordinary differential equations with generalized homogenous right-hand sides," Izvestia vuzov. Mathematica., vol. 1, pp. 80-88, 1958. in Russian.

[10] M. Kawski, "Nilpotent Lie algebras of vector fields," J. reine angew. Math, p. 1, 1988.

[11] L. Rosier, "Homogeneous Lyapunov function for homogeneous continuous vector field," Systems \& Control Letters, vol. 19 , pp. 467-473, 1992.

[12] H. Hermes, "Homogeneous feedback controls for homogeneous systems," Systems \& Control letters, vol. 24, pp. 7-11, 1995.

[13] R. Sepulchre and D. Aeyels, "Stabilizability does not imply homogeneous stabilizability for controllable systems," SIAM J. Control Optimization, vol. 34, no. 5, pp. 1798-1813, 1996.

[14] L. Grüne, "Homogeneous state feedback stabilization of homogeneous systems," SIAM J. Control Optimization, vol. 38, no. 4, pp. 1288-1314, 2000.

[15] S. Bhat and D. Bernstein, "Geometric homogeneity with applications to finite-time stability," Mathematics of Control, Signals and Systems, vol. 17, pp. 101-127, 2005.

[16] V. Bokharaie, O. Mason, and M. Verwoerd, "D-stability and delay-independent stability of homogeneous cooperative systems," Automatic Control, IEEE Transactions on, vol. 55, no. 12, pp. 2882-2885, 2010.

[17] A. Aleksandrov, A. Kosov, and A. Platonov, "On the asymptotic stability of switched homogeneous systems," Systems \& Control Letters, vol. 61, no. 1, pp. 127-133, 2012.

[18] V. Andrieu, L. Praly, and A. Astolfi, "Homogeneous Approximation, Recursive Observer Design, and Output Feedback," SIAM J. Control Optimization, vol. 47, no. 4, pp. 1814-1850, 2008.

[19] D. Efimov and W. Perruquetti, “Oscillations conditions in homogenous systems," in Proc. IFAC NOLCOS Symp., pp. 1379-1384, 2010.

[20] Y. Hong, "H $\mathrm{H}_{\infty}$ control, stabilization, and input-output stability of nonlinear systems with homogeneous properties," Automatica, vol. 37, no. 7, pp. 819$829,2001$.

[21] E. Ryan, "Universal stabilization of a class of nonlinear systems with homogeneous vector fields," Systems \& Control Letters, vol. 26, pp. 177-184, 1995.

[22] A. Bacciotti and L. Rosier, Lyapunov Functions and Stability in Control Theory. Springer, 2nd ed., 2005.

[23] H. Hermes, "Nilpotent approximations of control systems and distributions," SIAM J. Control Optim., vol. 24, p. 731, 1986.

[24] C. Ning, Y. He, M. Wu, Q. Liu, and J. She, "Input-to-state stability of nonlinear systems based on an indefinite lyapunov function," Systems \& Control Letters, vol. 61, no. 12, pp. 1254-1259, 2012.

[25] S. Bhat and D. Bernstein, "Finite time stability of continuous autonomous systems," SIAM J. Control Optim., vol. 38, no. 3, pp. 751-766, 2000.

[26] E. Moulay and W. Perruquetti, "Lyapunov-based approach for finite time stability and stabilization," in Decision and Control, 2005 and 2005 European Control Conference. CDC-ECC '05. 44th IEEE Conference on, pp. 4742-4747, 2005.

[27] Y. Hong, Z. Jiang, and G. Feng, "Finite-time input-to-state stability and applications to finite-time control design," SIAM Journal on Control and Optimization, vol. 48, no. 7, pp. 4395-4418, 2010.

[28] E. Bernuau, W. Perruquetti, D. Efimov, and E. Moulay, "Finite-time output stabilization of the double integrator," in Decision and Control (CDC), 2012 IEEE 51st Annual Conference on, pp. 5906-5911, 2012.

[29] W. Perruquetti and S. Drakunov, "Finite time stability and stabilisation," in IEEE Conference on Decision and Control, no. 2, (Sydney, Australia), pp. 1894-1899, 2000.

[30] S. P. Bhat and D. Bernstein, "Continuous finite-time stabilization of the translational and rotational double integrator," IEEE Trans. Automat. Control, vol. 43, pp. 678-682, may 1998. 